\title{
Matrix cracking of fiber-reinforced ceramic composites in shear
}

\author{
Varun P. Rajan *, Frank W. Zok \\ Materials Department, University of California, Santa Barbara, CA 93106, USA
}

\section{A R T I C L E I N F O}

\section{Article history:}

Received 9 December 2013

Received in revised form

10 August 2014

Accepted 23 August 2014

Available online 6 September 2014

\section{Keywords:}

Fracture

Fiber-reinforced composite material

Constitutive behavior

C. Finite elements

A. Energy release rate

\begin{abstract}
A B S T R A C T
The mechanics of cracking in fiber-reinforced ceramic matrix composites (CMCs) under general loadings remains incomplete. The present paper addresses one outstanding aspect of this problem: the development of matrix cracks in unidirectional plies under shear loading. To this end, we develop a model based on potential energy differences upstream and downstream of a fully bridged steady-state matrix crack. Through a combination of analytical solutions and finite element simulations of the constituent stresses before and after cracking, we identify the dominant stress components that drive crack growth. We show that, when the axial slip lengths are much larger than the fiber diameter and when interfacial slip precedes cracking, the shear stresses in the constituents are largely unaffected by the presence of the crack; the changes that do occur are confined to a 'core' region within a distance of about one fiber diameter from the crack plane. Instead, the driving force for crack growth derives mainly from the axial stresses-tensile in the fibers and compressive in the matrix-that arise upon cracking. These stresses are wellapproximated by solutions based on shear-lag analysis. Combining these solutions with the governing equation for crack growth yields an analytical estimate of the critical shear stress for matrix cracking. An analogous approach is used in deriving the critical stresses needed for matrix cracking under arbitrary in-plane loadings. The applicability of these results to cross-ply CMC laminates is briefly discussed.
\end{abstract}

(c) 2014 Elsevier Ltd. All rights reserved.

\section{Introduction}

A constitutive model for the inelastic mechanical response of fiber-reinforced ceramic composites must account for the anisotropy associated with the fiber architecture (Cady et al., 1995; Genin and Hutchinson, 1997). In two-dimensional layups, important insights into the mechanics of damage and deformation can be obtained by first considering separately the response of the constituent unidirectional plies and then addressing the interactions that occur between plies in the laminate. With respect to ply response, the key inelastic properties are those measured in tension and in shear parallel to the fiber axis. A large body of literature exists regarding the tensile response of such plies (Beyerle et al., 1992b; Curtin, 1991; Hui et al., 1995; Evans et al., 1994; Evans and Zok, 1994); much less attention has been directed to their shear response. The latter is the focus of the present paper.

\footnotetext{
* Corresponding author.

E-mail address: varun_rajan@umail.ucsb.edu (V.P. Rajan).
} 
The understanding of the axial tensile behavior of unidirectional ceramic composites is as follows. Initially the composite responds elastically with a modulus well described by the rule of mixtures: $E_{c}=V_{f} E_{f}+V_{m} E_{m}$, where $V$ is volume fraction, $E$ is Young's modulus, and subscripts $f$ and $m$ refer to fiber and matrix, respectively. At a critical stress, matrix cracks form and propagate long distances transverse to the tensile axis. The cracks are bridged by intact fibers and hence do not lead to catastrophic fracture. The stress needed to grow a steady-state, fully bridged matrix crack was derived from energy arguments in the seminal paper of Aveston et al. (1971). The model was later extended by Marshall et al. (1985), Budiansky et al. (1986), and Marshall and Cox (1988) to account for effects of non-zero interface debond toughness and residual stress. Interactions between neighboring matrix cracks through overlap of their respective slip zones and their effects on the evolution of crack density were addressed by Zok and Spearing (1992). Yet other features of the cracking process, including effects of flaw size and unbridged crack segments, have also been analyzed (Marshall et al., 1985; Spearing and Zok, 1993). Upon further loading, the crack density increases and eventually reaches saturation with an average spacing of, typically, (5-20) $d$, where $d$ is the fiber diameter. Thereafter the matrix bears no additional load and the composite tangent modulus approaches that of the fibers alone $\left(\mathrm{d} \sigma / \mathrm{d} \varepsilon \approx V_{f} E_{f}\right)$. Additional non-linearity is obtained once the fibers begin to break. Exact solutions for bundle fragmentation in the case where the fibers are frictionally coupled to a cracked matrix were developed by Hui et al. (1995) and useful approximations for bundle strength were derived by Curtin (1991).

Essentially the same failure mechanisms are operative in cross-ply laminates in uniaxial tension and hence similar mechanics analyses can be employed to describe the composite response. But here two additional features arise. First, tensile cracking of the transverse plies is usually the first inelastic event. Once the length of a transverse ply crack exceeds the ply thickness, the crack grows by 'tunneling' between adjacent axial plies under steady state conditions (i.e., with constant energy release rate, independent of crack length) (Beyerle et al., 1992a; Xia et al., 1993). Furthermore, multiple cracks are formed in each ply, eventually saturating when their spacing becomes comparable to the ply thickness. Second, at higher stresses, the cracks penetrate the axial plies. Because the segments of the cracks within the transverse plies are unbridged, the stress needed for full penetration into the axial plies is lower than the steady state matrix cracking stress in unidirectional materials (adjusting the stress to account for the reduced fraction of axial plies) (Xia and Hutchinson, 1994). The fiber fragmentation process that controls ultimate strength is largely unaffected by the presence of the transverse plies, apart from their effects on the volume fraction of fibers aligned with the loading direction (Beyerle et al., 1992a).

A comparable level of understanding of the shear response of cross-ply CMC laminates is presently lacking. Limited experimental studies (Brøndsted et al., 1994; Cady et al., 1995; Turner et al., 1995) have shown that the principal damage mechanism involves matrix cracking at approximately $45^{\circ}$ to the fibers, i.e., perpendicular to the direction of maximum principal stress. As in tension, the cracks are initially fully bridged by intact fibers and propagate long distances without catastrophic fracture. The average crack spacing at saturation is approximately (5-10)d. For cross-ply laminates with dense matrices, typical values of matrix cracking stress in shear are comparable to those in tension. (The cracking stress in tension for a unidirectional material with the same fiber volume fraction is significantly higher, for reasons mentioned previously.) In shear, as in tension, the matrix cracking stress increases with matrix stiffness. For the specific example of SiC/CAS (Brøndsted et al., 1994), matrix cracking ultimately saturates at a strain of roughly $1 \%:^{1}$ only slightly below the corresponding fracture strain. This observation suggests the absence of a fiber-dominated regime such as that observed in uniaxial tension. For cross-ply laminates, failure strains in shear are somewhat larger than those in tension and are inversely related to matrix stiffness. Brøndsted et al. (1994) attempted to explain the latter observation by considering local bending of fibers at matrix cracks, although a rigorous mechanistic understanding of the phenomenon was lacking in that work.

The principal objective of the present study is to develop the mechanics underlying the onset of matrix cracking in fiberreinforced ceramic composites under shear loading. The study is restricted to unidirectional materials, with the understanding that some modification may be required to adapt the results to multidirectional laminates. The focus is on the stress distributions in the fibers and matrix before and after matrix cracking. The corresponding difference in potential energy is used to determine the shear stress for cracking under steady-state conditions.

The outline of the paper is as follows. First, the governing equation, adapted from the work of Budiansky et al. (1986), is presented for steady-state matrix cracking in shear. The driving force for crack growth derives from differences in stresses in the uncracked and cracked states. Second, the stresses in the uncracked composite are obtained from an analytical model of a cylindrical unit cell, extending solutions previously developed by Hashin and Rosen (1964). Third, the stresses in the cracked composite are obtained from finite element (FE) analyses of a representative volume element (RVE). The model employs periodic boundary conditions that ensure stress and displacement continuity between adjacent RVEs. Fourth, the results of the FE model are used to guide the development of analytical solutions (based on shear-lag analysis) for fiber and matrix stresses in a cracked composite. Then, using the analytical solutions for the dominant stresses within the fibers and the matrix in the cracked and uncracked states, expressions are derived for the matrix cracking stress. The cracking stresses in shear and tension (Aveston et al., 1971; Budiansky et al., 1986) are found to exhibit similar (though not identical) scalings with the constituent properties. Finally, the results are generalized to the case of arbitrary in-plane loading (both tension and shear).

\footnotetext{
${ }^{1}$ All shear strains referred to herein are engineering shear strains.
} 
a

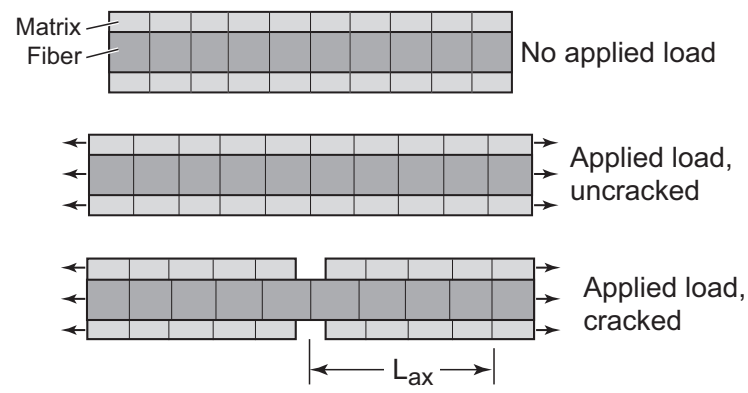

b

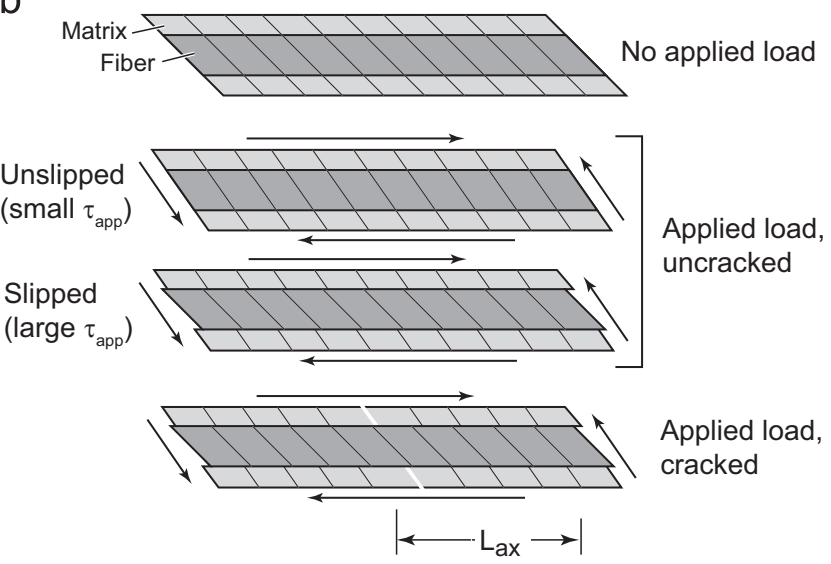

Fig. 1. Development of frictional slip in a unidirectional CMC in (a) tension and (b) shear.

As a prelude to the results that follow, we highlight two key differences between the shear cracking problem considered here and the tensile cracking problem addressed by Aveston et al. (1971) and Budiansky et al. (1986). The discussion is restricted to cases where the fiber/matrix interfaces are initially unbonded but frictionally coupled. (Additional complications arise if the interfaces are initially bonded.) Accompanying schematic illustrations are shown in Fig. 1. (i) In tension, prior to cracking, there is no driving force for interfacial separation or slip and thus the material response is purely elastic: obtaining stresses and strain energies for this state is trivial. In contrast, when the composite is loaded in shear, the fibermatrix interface experiences a shear stress that varies linearly across the fiber diameter: the maximum occurring at the two 'poles' and zero stress occurring along the fiber mid-plane. This raises the possibility of interfacial slip prior to matrix cracking. Furthermore, the non-uniformity of the shear stresses adds complexity to the prediction of the slip processes. (ii) In tension, after cracking, frictional slip occurs in an axisymmetric manner, significantly simplifying the stress analysis. In contrast, in shear, frictional slip manifests itself in two distinct ways. In the first, termed 'antisymmetric slip', the average axial displacements of the fiber and the matrix are identical. (By 'average', we mean that the quantity is integrated over the interface at a particular value of the axial coordinate $x$ ). In this case, the matrix slips positively relative to the fiber along one half of the interface and negatively with respect to the fiber along the other half. In the second, termed 'axial slip', the average axial displacements of the fiber and the matrix differ. We show below that this occurs as a consequence of the tensile stresses developed within the fiber after matrix cracking in shear.

\section{Matrix cracking model}

The critical stress for steady-state matrix cracking in shear is derived using a fracture mechanics-based approach, closely analogous to that presented by Budiansky et al. (1986) for matrix cracking in axial tension. The driving force for crack growth is the potential energy difference between the cracked and uncracked segments; crack growth occurs when this energy difference is equal to the fracture resistance. Computation of the potential energy difference requires stresses in the 'upstream' and the 'downstream' locations with respect to the crack tip.

The fibers are assumed to be frictionally coupled but initially unbonded from the matrix (i.e., the interfacial toughness is zero). We show below that, in cases where the conditions for frictional slip are met in the uncracked state, the introduction of a matrix crack leaves the shear stresses in the fibers and the matrix largely unaffected. However, the presence of the matrix crack creates axial tension in the fibers and axial compression in the matrix. The differences in these stresses provide the driving force for cracking; the corresponding resistance is the energy needed to create the matrix crack surfaces. The governing equation for steady-state growth of a shear crack is found through an adaptation of Equation 11 in 
Budiansky et al. (1986), notably:

$$
\frac{1}{2 A_{c}} \int_{-\infty}^{\infty} \int_{A_{c}}\left(\boldsymbol{\sigma}^{U}-\boldsymbol{\sigma}^{D}\right):\left(\boldsymbol{\varepsilon}^{U}-\boldsymbol{\varepsilon}^{D}\right) \mathrm{d} A \mathrm{~d} x=\sqrt{2} V_{m} \mathcal{G}_{m}
$$

Here, $\boldsymbol{\sigma}$ and $\boldsymbol{\varepsilon}$ are stress and strain tensors, respectively, in the upstream $(U)$ and downstream $(D)$ locations. The area integral is taken over a representative cross-sectional area of the composite, $A_{c}$, comprising a fiber and the surrounding matrix jacket. The $x$-direction is aligned with the fiber axis, $\mathcal{G}_{m}$ is the fracture energy of the matrix, and the factor $\sqrt{2}$ accounts for the fact that the crack is oriented at $45^{\circ}$ to the fiber axis. Eq. (1) assumes that no point on the fiber-matrix interface experiences variable-direction slip between the upstream and downstream states, and that the slip process at each point occurs at a constant sliding stress.

As demonstrated in a forthcoming section, the upstream stresses can be well approximated using an analytical model of a cylindrical unit cell (Section 4). In contrast, the downstream stresses are not amenable to a fully analytical solution. Consequently, finite element analyses (Section 3) are used to generate numerical results that are then used to guide the formulation of semi-analytical solutions that capture the dominant stress components (Section 5). The stresses are then combined with Eq. (1) in order to ascertain the steady-state matrix cracking stress (Sections 6 and 7).

\section{Finite element model}

\subsection{Geometry and mesh}

One crucial consideration in computational micromechanical modeling is the fidelity with which heterogeneities in microstructure are captured. In laminates undergoing shear loading, the fibers and the matrix cracks are generally distributed non-uniformly. Capturing these heterogeneities requires that at least tens of fibers and several matrix cracks comprise the RVE; the minimum size of the resulting RVE for a typical composite with fiber diameter $d=10 \mu \mathrm{m}$ would be approximately $100 \mu \mathrm{m} \times 100 \mu \mathrm{m} \times 1 \mathrm{~mm}$. Assuming that an element size of $d / 10$ is necessary to properly resolve local stresses, a conservative estimate of the number of elements within such a model is $10^{7}$ : beyond that which is computationally feasible. (Note that this situation contrasts markedly with RVE models used to compute the deformation response of polymer matrix composites (PMCs). In PMCs undergoing plastic deformation, the length of the RVE can be taken to be arbitrarily small, thereby allowing for a large number of fibers with non-uniform spacing to be readily tackled with existing computational capabilities (see, for instance, Totry et al., 2010).)

In order to strike a balance between computational efficiency and model accuracy, we assume here that the fibers are arranged in a regular, square array and that the matrix cracks are evenly spaced. The RVE thus becomes a parallelepiped unit cell, composed of a single fiber enclosed in a cracked matrix jacket (Fig. 2). The fiber axis is parallel to the $x$-direction, and the normal to the crack plane lies in the $x-y$ plane, at $45^{\circ}$ with respect to the $x$-axis. The unit cell is tiled in space in a staggered manner to generate the entire unidirectional composite. The two cell dimensions transverse to the fiber axis, $L_{y}=L_{z}$, are equivalent to the center-to-center fiber spacing and the fiber volume fraction is related to the dimensions by $V_{f}=\pi d^{2} / 4 L_{y}^{2}$. The third RVE dimension, $L_{x}$, represents the matrix crack spacing. In principle, the matrix crack can be positioned anywhere within the RVE; we locate it centrally to preserve the antisymmetry of the deformation. The origin of the coordinate system defining material locations is taken to be the intersection of the matrix crack plane with the fiber axis.

Finite element simulations were conducted in ABAQUS Standard (Version 6.9-EF1, Dassault Systèmes). The mesh was carefully constructed to enable application of periodic boundary conditions (Section 3.2). Specifically, when a node exists on the boundary of the RVE, all points equivalent to it-i.e., points that are periodically related to it-must also exist as nodes on the boundary of the RVE. This condition was operationally realized using the following procedure. First, a 2-D mesh was generated of a fiber enclosed by a matrix jacket. In the 2-D mesh, both the left and right edges as well as the top and bottom edges contained periodic pairs of nodes. Furthermore, the fiber and matrix nodes were constructed to be coincident at the fiber-matrix interface; this choice was found to greatly reduce numerical oscillations in contact pressure along the interface. The mesh was then transformed to create the $\left(45^{\circ}\right)$ right face of the unit cell, and was swept in the $x$-direction to generate the entire parallelepiped. Because the mesh is swept, the 4-noded elements that comprise the 2-D mesh become 8-noded bricks within the 3-D mesh. Finally, the matrix crack was inserted by dividing the matrix jacket into two separate parts.

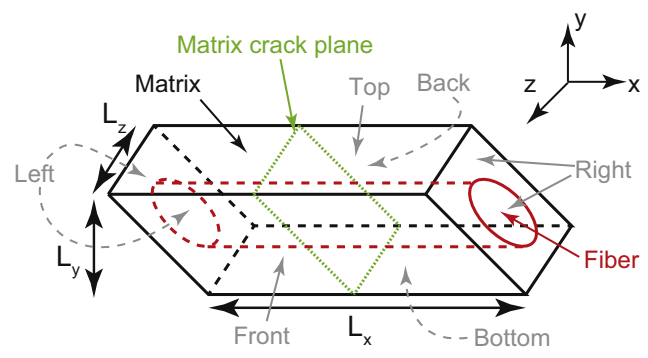

Fig. 2. Parallelepiped unit cell used in finite element model. 
A typical finite element simulation used first-order, reduced integration elements (C3D8R) with a characteristic length of 5\% of the fiber diameter; for typical matrix crack spacings (e.g. $L_{x} / d=10$ ), the total number of elements within the model was approximately 120,000 . For a limited number of representative simulations, a study was performed to ensure that the quantities of interest (e.g. fiber bending stress) converged with respect to mesh density.

\subsection{Boundary conditions}

The boundary conditions were designed to achieve two objectives. First, they must enforce stress and displacement continuity between adjacent RVEs. Second, they must create the desired state of stress within the RVE. The stress state is imposed only in the averaged sense - that is, the volume average of stresses within the RVE. Here, we attempt to achieve a state of shear stress within the RVE, expressed mathematically as

$$
\begin{aligned}
& \hat{\sigma}_{x y}=\hat{\sigma}_{y x}=\tau_{a p p} \\
& \hat{\sigma}_{i j}=0 \quad i, j=\text { other }
\end{aligned}
$$

where $\hat{\sigma}_{i j}$ is the tensor of average stresses within the RVE.

Both objectives are met by utilizing the periodicity condition (Suquet, 1987; Xia et al., 2003). Displacements on the boundary of a RVE are decomposed into two parts: a systematic component, which is related to the applied strain field, and a periodic component, which is generally unknown. The condition can be expressed as (Suquet, 1987; Xia et al., 2003)

$$
u_{i}=\hat{\varepsilon}_{i k} x_{k}+u_{i}^{*}
$$

where $u_{i}$ is the displacement of the point in the $i$-direction, $\hat{\varepsilon}_{i k}$ is the tensor of average applied strains, $x$ is the position of the point, and $u_{i}^{*}$ is the periodic component of displacement in the $i$-direction. For points within the RVE that are equivalent (i.e., periodically related to one another), $u_{i}^{*}$ must be identical. This ensures that the deformations of neighboring RVEs are compatible. By considering differences in displacements between equivalent points, the periodic component of displacement cancels out, which results in a simple relationship between the displacement difference and the applied strain.

The periodic boundary conditions considered here are not, in general, equivalent to either uniform displacement or uniform traction boundary conditions. Periodic boundary conditions enforce displacements and tractions along the boundary of the RVE in an average sense, not in a pointwise sense. In general, using uniform displacement or traction boundary conditions violates stress periodicity, as discussed by Xia et al. (2003).

To formulate the displacement difference for the parallelepiped unit cell, we consider the three pairs of surfaces in the unit cell that are equivalent/periodic (see Fig. 2(b)): (i) the right $(R)$ and left $(L F)$ surfaces of the fiber and matrix; (ii) the top $(T)$ and bottom $(B M)$ surfaces of the matrix; and (iii) the front $(F)$ and back $(B K)$ surfaces of the matrix. For surface pair (i), we obtain the expressions

$$
\begin{aligned}
& u_{x}(R)-u_{x}(L F)=\hat{\varepsilon}_{x x} L_{x} \\
& u_{y}(R)-u_{y}(L F)=\hat{\varepsilon}_{y x} L_{x} \\
& u_{z}(R)-u_{z}(L F)=\hat{\varepsilon}_{z x} L_{x}
\end{aligned}
$$

Similarly, for surface pair (ii),

$$
\begin{aligned}
& u_{x}(T)-u_{x}(B M)=-\hat{\varepsilon}_{x x} \Delta x+\hat{\varepsilon}_{x y} L_{y} \\
& u_{y}(T)-u_{y}(B M)=-\hat{\varepsilon}_{y x} \Delta x+\hat{\varepsilon}_{y y} L_{y} \\
& u_{z}(T)-u_{z}(B M)=-\hat{\varepsilon}_{z x} \Delta x+\hat{\varepsilon}_{z y} L_{y}
\end{aligned}
$$

where $\Delta x=L_{y}$ since the left and right surfaces are oriented at $45^{\circ}$ to the $x$-axis.

Ideally, the boundary conditions would be written so that either average strains or average stresses could be prescribed. In their current form, Eqs. (4) and (5) allow only average strains to be imposed. To correct this deficiency, three 'fictitious' nodes, denoted $F x, F y$, and $F z$, are constructed. The nodes are fictitious in the sense that they are not part of either the fiber or matrix meshes. Instead, they are connected to 'real' nodes on the RVE boundary through constraint equations. A length, $L(F j)=L_{j}$, and an area, $A(F j)=V / L_{j}$, are associated with each fictitious node $F j$ (where $V=L_{x} L_{y} L_{z}$ ). Each strain component is written in terms of the displacement of a fictitious node, $u(F j)$ :

$$
\hat{\varepsilon}_{i j}=\frac{u_{i}(F j)}{L(F j)}
$$

Combining with Eq. (5), for instance, yields

$$
\begin{aligned}
& u_{x}(T)-u_{x}(B M)=-\frac{\Delta x}{L_{x}} u_{x}(F x)+u_{x}(F y) \\
& u_{y}(T)-u_{y}(B M)=-\frac{\Delta x}{L_{x}} u_{y}(F x)+u_{y}(F y) \\
& u_{z}(T)-u_{z}(B M)=-\frac{\Delta x}{L_{x}} u_{z}(F x)+u_{z}(F y)
\end{aligned}
$$


Similarly, each stress component is written in terms of the force applied to a fictitious node, $F(F j)$ :

$$
\hat{\sigma}_{i j}=\frac{F_{i}(F j)}{A(F j)}
$$

To impose an average strain, a displacement is applied to the appropriate fictitious node according to Eq. (6); to impose an average stress, a force is applied according to Eq. (8). In the case of zero average stress, zero force is applied to the fictitious node: the node relaxes to ensure that the net reaction force is zero. The fictitious node approach is versatile in that a mixture of stress and strain conditions can be enforced. In the case of shear loading considered here, all fictitious nodes corresponding to stress components apart from $\hat{\sigma}_{x y}\left(=\hat{\sigma}_{y x}\right)$ are allowed to relax, so that the associated average stress components are zero.

\subsection{Constitutive behavior}

The fibers and matrix were assumed to be linear elastic and isotropic with a Poisson's ratio of 0.2 (a value representative of ceramics). Two surface interactions were specified: that between the matrix crack faces (when present) and that between the fiber and the matrix.

The matrix crack faces were allowed to undergo frictionless slip in the tangential direction and were subjected to "hard contact' in the normal direction. (As a practical matter, we find that the crack faces separate during shear loading and thus the results are unaffected by the prescribed contact interaction.)

Frictional slip between the fiber and the matrix was intended to occur at a constant interfacial sliding stress $\tau_{s}$, independent of the normal compression across the interface, as in the works of Aveston et al. (1971) and Curtin (1991) (whether this assumption is physically realistic is addressed in Section 8). However, for convenience, the computational implementation of this interaction (in ABAQUS) utilized the built-in Coulomb friction law with a shear stress 'cap'. Specifically, when the interface is closed, with interfacial pressure $p>0$, either a 'sticking' or a 'slip' condition obtains, depending on the interfacial shear stress. The critical shear stress for slip is defined as

$$
\tau_{c r}=\min \left(\mu p, \tau_{s}\right)
$$

where $\mu$ is the friction coefficient and $\tau_{s}$ is the desired (constant) sliding stress. When the equivalent shear stress ${ }^{2}$ along the interface exceeds $\tau_{c r}$, the fiber slides relative to the matrix and the sliding stress along the interface is equal to $\tau_{c r}$. The friction coefficient $\mu$ is selected to be sufficiently large to achieve slip at the desired stress $\tau_{s}$. (Conversely, if $\tau_{e q}<\tau_{c r}$, then no slip occurs along the interface: the 'sticking' condition obtains.)

For slip to occur in the manner intended, a positive pressure must be present across the interface between the fiber and the matrix. (No interaction occurs between the fiber and matrix when the interface is open.) Operationally, this condition was effected by specifying a mismatch in thermal expansion coefficients of the two constituents, and subsequently imposing an isothermal temperature change that yielded the desired average interfacial pressure, $\hat{p}$. This process also yields residual axial compression in the fiber and residual axial tension in the matrix (in the $x$-direction). The latter stresses are on the order of $\hat{p}$ (Budiansky et al., 1986).

\section{Response of uncracked composite}

\subsection{Preliminaries}

Here we present solutions for the stress distributions in both phases and the global response of the uncracked composite under shear loading. We begin with formulae obtained from analyses of a cylindrical unit cell (Hashin and Rosen, 1964). The diameter of the matrix jacket in this cell is taken as $d_{m}=d / \sqrt{V_{f}}$. Displacements (in cylindrical coordinates) are taken to have the form

$$
\begin{aligned}
& u_{r}=C x \sin \theta \\
& u_{\theta}=C x \cos \theta \\
& u_{x}=\left(A r+\frac{B}{r}\right) \sin \theta
\end{aligned}
$$

All stress components are zero, except

$$
\begin{aligned}
\sigma_{x \theta} & =G\left(A+C+\frac{B}{r^{2}}\right) \cos \theta \\
\sigma_{x r} & =G\left(A+C-\frac{B}{r^{2}}\right) \sin \theta
\end{aligned}
$$

\footnotetext{
${ }^{2}$ The equivalent shear stress is $\tau_{e q}=\sqrt{\tau_{x r}^{2}+\tau_{\theta r}^{2}}$, where $\tau_{x r}$ and $\tau_{\theta r}$ are the components of shear stress in the two tangential directions along the interface. The results of the simulation demonstrate that $\tau_{\theta r}=0$ almost everywhere; $\tau_{e q}$ is dominated by the $\tau_{x r}$, the shear stress in the axial direction.
} 
where $G$ is the shear modulus of the appropriate phase. There are six unknown constants: $A, B$, and $C$ for each of the two phases. They are obtained by assigning appropriate boundary conditions. Continuity of radial displacement $\left(u_{r}\right)$ and shear stress $\left(\sigma_{x r}\right)$ must hold at the fiber-matrix interface $(r=d / 2)$. The shear stress $\sigma_{x r}$ at the external boundary $\left(r=d_{m} / 2\right)$ must equal the applied shear traction, which has the form $\tau_{a p p}^{0}(r, \theta)=\tau_{a p p} \sin \theta$. Also, the applied shear traction must be in equilibrium with the resultant of the shear stresses along a plane of constant $x$. The condition for axial displacements $\left(u_{x}\right)$ at the fiber-matrix interface depends on the state of the interface. That is, when the applied stress is sufficiently small, the interface remains unslipped and the axial displacements in the fiber and the matrix are equal. The solutions for this scenario are presented in Section 4.2. Conversely, when the applied stress is large, antisymmetric slip occurs between the fiber and the matrix, and the condition of continuity of $u_{x}$ is no longer met. The pertinent solutions are presented in Section 4.3. The analytical predictions are assessed through comparisons with results from finite element solutions for several cases of practical interest.

With the stresses and strains in hand, the tangent modulus of the unit cell is derived using the principle of virtual work. All stresses, strains and displacements are expressed in terms of the applied shear load $P$ (for a unit cell in shear, $\left.P=\pi d_{m}^{2} \tau_{a p p} / 4\right)$. The statement reads

$$
\int_{V} \boldsymbol{\sigma}(P): \delta \boldsymbol{\varepsilon}(P) \mathrm{d} V+\int_{S} \tau \delta \Delta u_{\tau}(P) \mathrm{d} S=P \delta u_{P}(P)
$$

where $\Delta u_{\tau}$ is the difference in axial $\left(u_{x}\right)$ displacement between the fiber and the matrix, and $u_{P}$ is the shear displacement of the cell (i.e., the work conjugate to $P$ ).

Using the chain rule,

$$
\int_{V} \boldsymbol{\sigma}(P): \frac{\mathrm{d} \boldsymbol{\varepsilon}(P)}{\mathrm{d} P} \delta P \mathrm{~d} V+\int_{S} \tau \frac{\mathrm{d} \Delta u_{\tau}(P)}{\mathrm{d} P} \delta P \mathrm{~d} S=P \frac{\mathrm{d} u_{P}(P)}{\mathrm{d} P} \delta P
$$

Since $\delta P$ is arbitrary, we obtain

$$
\frac{\mathrm{d} \tau_{a p p}}{\mathrm{~d} \gamma}=\frac{4 L_{x}}{\pi d_{m}^{2}} \frac{\mathrm{d} P}{\mathrm{~d} u_{P}}=\frac{4 L_{x}}{\pi d_{m}^{2}} \frac{P}{\int_{V} \sigma(P): \frac{\mathrm{d} \boldsymbol{\varepsilon}(P)}{\mathrm{d} P} \mathrm{~d} V+\int_{S} \tau \frac{\mathrm{d} \Delta u_{\tau}(P)}{\mathrm{d} P} \mathrm{~d} S}
$$

\subsection{Elastic response}

For small applied stresses, the composite response is elastic. In this scenario, analytical solutions for the constants $A, B$, and $C$ (previously presented by Hashin and Rosen, 1964) are

$$
\begin{aligned}
A_{m} & =\frac{\tau_{a p p}\left(G_{m}+G_{f}\right)}{G_{m}\left(G_{m} V_{m}+G_{f}\left(1+V_{f}\right)\right)}-C_{0} \\
B_{m} & =\frac{d^{2} \tau_{a p p}\left(G_{m}-G_{f}\right)}{4 G_{m}\left(G_{m} V_{m}+G_{f}\left(1+V_{f}\right)\right)} \\
A_{f} & =\frac{2 \tau_{a p p}}{G_{m} V_{m}+G_{f}\left(1+V_{f}\right)}-C_{0} \\
B_{f} & =0 \\
C_{f} & =C_{m}=C_{0}
\end{aligned}
$$

where $C_{0}$ is an unknown constant related to rigid body rotation that does not affect the stresses. Combining with Eq. (11) yields the pertinent stresses:

$$
\begin{aligned}
& \sigma_{x r, f}=\frac{2 G_{f}}{G_{m} V_{m}+G_{f}\left(1+V_{f}\right)} \tau_{a p p} \sin \theta \\
& \sigma_{x \theta, f}=\frac{2 G_{f}}{G_{m} V_{m}+G_{f}\left(1+V_{f}\right)} \tau_{a p p} \cos \theta \\
& \sigma_{x r, m}=\frac{G_{m}\left(1-(d / 2 r)^{2}\right)+G_{f}\left(1+(d / 2 r)^{2}\right)}{G_{m} V_{m}+G_{f}\left(1+V_{f}\right)} \tau_{a p p} \sin \theta \\
& \sigma_{x \theta, m}=\frac{G_{f}\left(1-(d / 2 r)^{2}\right)+G_{m}\left(1+(d / 2 r)^{2}\right)}{G_{m} V_{m}+G_{f}\left(1+V_{f}\right)} \tau_{a p p} \cos \theta
\end{aligned}
$$

Further combining these results with Eq. (14) recovers the result for the shear modulus from the composite cylinder assemblage (CCA) model (Hashin, 1983):

$$
G_{x y}=\frac{\mathrm{d} \tau_{a p p}}{\mathrm{~d} \gamma}=G_{m} \frac{G_{f}\left(1+V_{f}\right)+G_{m} V_{m}}{G_{f} V_{m}+G_{m}\left(1+V_{f}\right)}
$$

Comparisons of stress distributions for one specific case are shown in Fig. 3. Very good agreement is obtained within the entire fiber and over most of the matrix volume; differences naturally arise at the cell boundaries because of the differing 

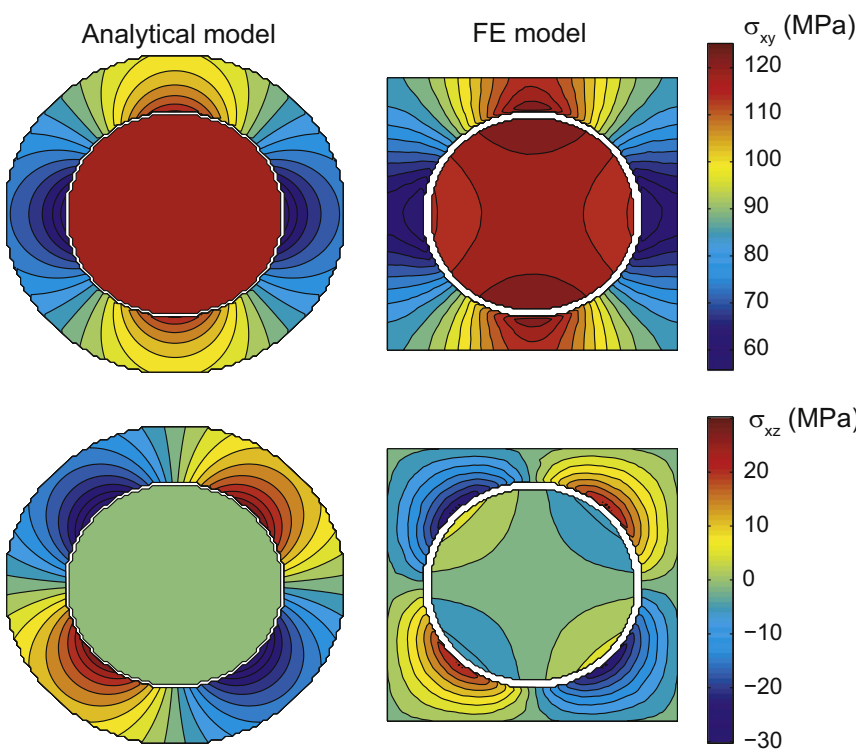

Fig. 3. Contour plots of $\sigma_{x y}$ and $\sigma_{x z}$ from the analytical model (cylindrical unit cell) and the finite element model for the uncracked, elastic composite $\left(V_{f}=0.4, E_{f}=210 \mathrm{GPa}, E_{m}=100 \mathrm{GPa}, \hat{p}=50 \mathrm{MPa}\right.$, and $\left.\tau_{a p p}=100 \mathrm{MPa}\right)$.
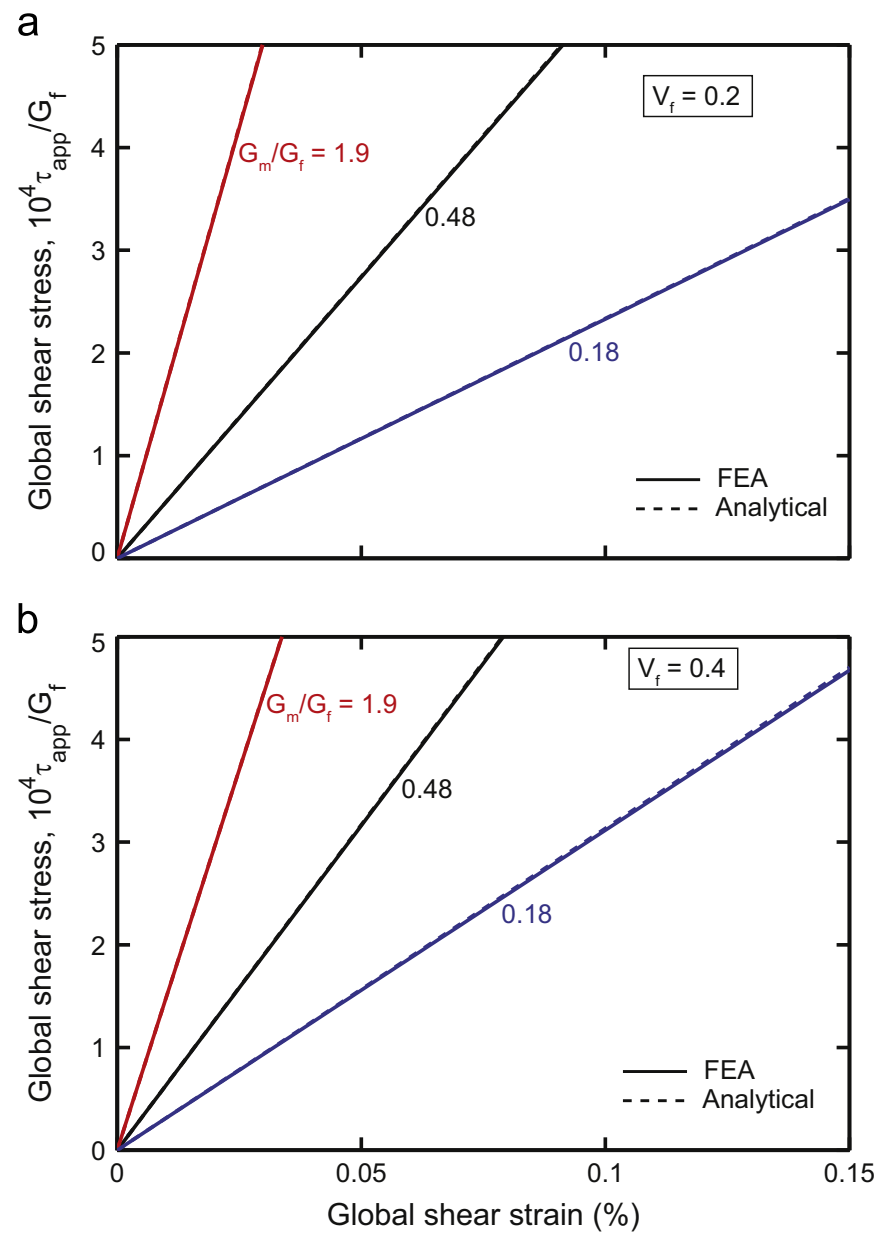

Fig. 4. Analytical predictions and finite element results of the globalstress-strain behavior of the uncracked, elastic composite for various values of matrix modulus and fiber volume fraction $\left(E_{f}=210 \mathrm{GPa}\right.$ and $\left.\hat{p}=50 \mathrm{MPa}\right)$. 
cell cross-sections (circular vs. square). Moreover, stress components predicted to be zero by the analytical model ( $\sigma_{x x}, \sigma_{y y}$, $\sigma_{z z}$, and $\sigma_{y z}$ ) are indeed very small (after subtracting the residual stresses) in the finite element simulations (not shown). Analogous comparisons for other material property combinations yielded similarly good agreement.

Comparisons of the global shear response are presented in Fig. 4. Here the fiber is assumed to be ceramic grade Nicalon ( $210 \mathrm{GPa}$ ), the fiber volume fraction is 0.2 or 0.4 , and the matrix modulus $E_{m}$ is selected to be representative of one of three common matrix materials: $37 \mathrm{GPa}$ for carbon, $100 \mathrm{GPa}$ for glass-ceramics, and $400 \mathrm{GPa}$ for SiC. For all cases, the analytical predictions and the computational results agree to within $0.6 \%$. The agreement indicates that the difference in the unit cell geometry in the finite element and analytical models is inconsequential for prediction of macroscopic properties.

\subsection{Inelastic response}

For sufficiently large applied stresses, the composite response is inelastic because antisymmetric slip occurs between the fiber and matrix. The condition of continuity of $u_{x}$ at $r=d / 2$ is replaced by the condition that the shear stress $\sigma_{x r}$ at $r=d / 2$ must equal the frictional sliding stress, which must take the form $\tau(r, \theta)=\tau_{s 2} \sin \theta$. To maintain consistency with the FE model, where $|\tau(r, \theta)|=\tau_{s}$ everywhere, the effective sliding stress $\tau_{\mathrm{s} 2}$ is selected to be that which gives the equivalent frictional dissipation during sliding, notably $\tau_{s 2}=4 \tau_{s} / \pi$. Using these formulae yields

$$
\begin{aligned}
& A_{m}=\frac{\tau_{a p p}-\tau_{s 2} V_{f}}{G_{m} V_{m}}-C_{0} \\
& B_{m}=\frac{d^{2}\left(\tau_{a p p}-\tau_{s 2}\right)}{4 G_{m} V_{m}} \\
& A_{f}=\frac{\tau_{s 2}}{G_{f}}-C_{0} \\
& B_{f}=0 \\
& C_{f}=C_{m}=C_{0}
\end{aligned}
$$

Combining with Eq. (11) gives the stresses as

$$
\begin{aligned}
& \sigma_{x r, f}=\tau_{s 2} \sin \theta \\
& \sigma_{x \theta, f}=\tau_{s 2} \cos \theta \\
& \sigma_{x r, m}=\frac{\left(\tau_{a p p}-\tau_{s 2} V_{f}\right)-\left(\tau_{a p p}-\tau_{s 2}\right)(d / 2 r)^{2}}{V_{m}} \sin \theta
\end{aligned}
$$
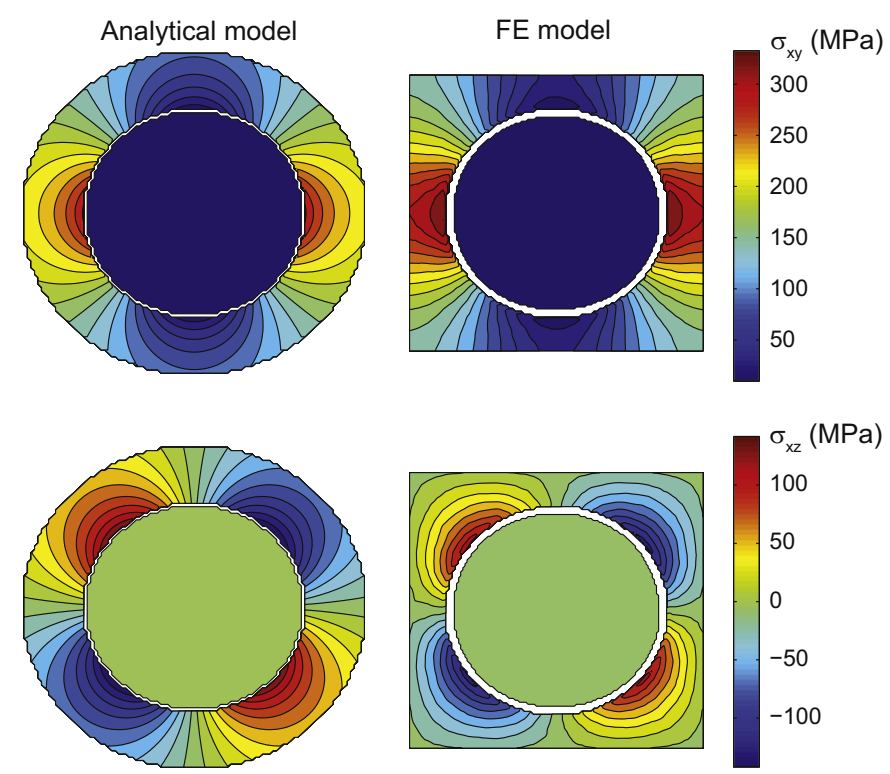

Fig. 5. Contour plots of $\sigma_{x y}$ and $\sigma_{x z}$ from the analytical model (cylindrical unit cell) and the finite element model for the uncracked, inelastic composite $\left(\tau_{a p p}=100 \mathrm{MPa}, V_{f}=0.4, E_{f}=210 \mathrm{GPa}, E_{m}=100 \mathrm{GPa}, \hat{p}=50 \mathrm{MPa}\right.$, and $\left.\tau_{s}=10 \mathrm{MPa}\right)$. 


$$
\sigma_{x \theta, m}=\frac{\left(\tau_{a p p}-\tau_{s 2} V_{f}\right)+\left(\tau_{a p p}-\tau_{s 2}\right)(d / 2 r)^{2}}{V_{m}} \cos \theta
$$

Additionally, the tangent modulus at large applied stresses becomes

$$
\frac{\mathrm{d} \tau_{a p p}}{\mathrm{~d} \gamma}=G_{m} \frac{V_{m}}{1+V_{f}}
$$

This is identical to the shear modulus predicted by the CCA model (Eq. (17)) when $G_{f} \rightarrow 0$. The applied stress, $\tau_{a p p}^{t r}$, at which the response transitions from Eq. (17)-(20) can be derived by equating the interfacial shear stress for the unslipped composite to the shear sliding stress. Doing so yields

$$
\tau_{a p p}^{t r}=\frac{G_{f}\left(1+V_{f}\right)+G_{m} V_{m}}{2 G_{f}} \tau_{s 2}
$$

For typical constituent properties, $\tau_{a p p}^{t r}$ is of the same order as $\tau_{s}$.

Comparisons of stress distributions in the fiber and the matrix for one case are shown in Fig. 5. Again, very good agreement is obtained between analytical predictions and computational results over most of the unit cell volume; differences arise at the cell boundaries because of differences in cell geometry. The predicted global responses (plotted in Fig. 6) are also in close agreement. Specifically, the tangent moduli in the high stress domain agree within $2 \%$ for all cases. The stress at which the behavior transitions from elastic to inelastic is also predicted fairly well, although the computational stress-strain curve is somewhat 'smeared' at the transition point. The latter occurs because the interfacial shear stress distribution must transition from the sinusoidal spatial variation to a step-like one over a finite strain range.
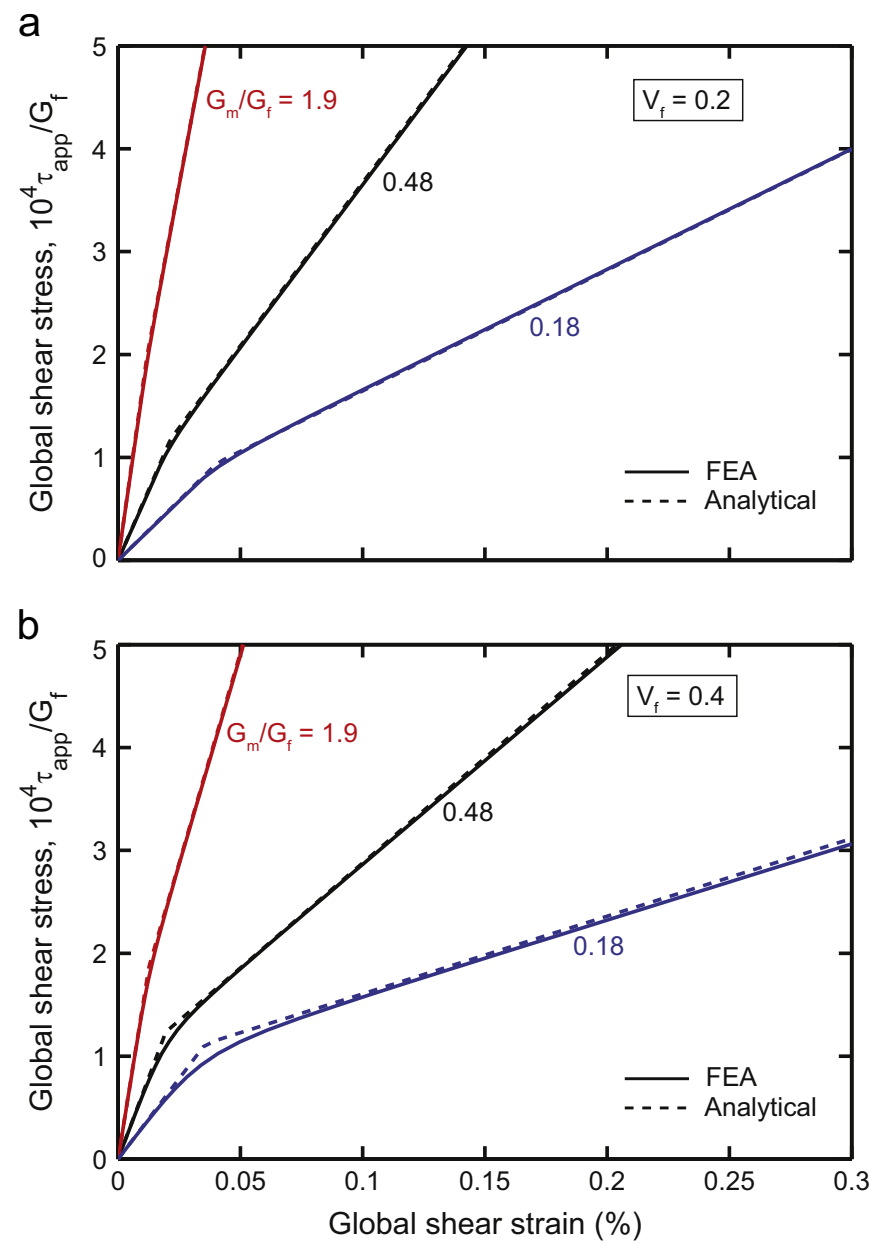

Fig. 6. Analytical predictions and finite element results of the global stress-strain behavior of the uncracked, inelastic composite for various values of matrix modulus and fiber volume fraction $\left(E_{f}=210 \mathrm{GPa}, \hat{p}=50 \mathrm{MPa}\right.$, and $\left.\tau_{s}=10 \mathrm{MPa}\right)$. 


\section{Response of cracked composite}

\subsection{Preliminaries}

No simple analytical model exists for the stresses in the cracked unit cell. Consequently, finite element simulations are employed to establish the form of the dominant stress components for cases of practical interest, and, in turn, to develop approximate analytical solutions for these stress components. The baseline FE simulation assumes a matrix crack spacing of $L_{x} / d=10$, which is large enough to differentiate the stresses arising from the presence of the matrix crack from the far-field stresses. The parameter values for this and other simulations are summarized in Table 1.

The principal result that arises from the FE simulations is that large bending and tensile stresses develop within the fiber adjacent to the matrix crack, as conjectured by Brøndsted et al. (1994). To illustrate, the distribution of $\sigma_{x x}$ within the fiber (at $z=0$ ) for the baseline simulation is shown in Fig. 7. The maximum tensile stress within the fiber, roughly 1.3 GPa, is more than an order of magnitude larger than the applied shear stress (100 MPa). Furthermore, the difference between the magnitude of stresses on the tensile and compressive faces indicates that a net tensile stress-again, significantly in excess of the applied shear stress-is also present.

\subsection{Free body analysis}

The origin of the tensile and bending stresses in the fibers can be understood through a two-dimensional free body analysis of the unit cell (Fig. 8(a)). (For convenience, the unit cell selected for this analysis differs slightly from the one depicted in Fig. 2, in that the matrix crack planes lie along the left and right surfaces rather than in the cell center.) The $R$ vectors are force resultants, obtained by integrating the traction over the surface. As discussed by Xia et al. (2003), the average stress within the unit cell, $\hat{\sigma}$, can be expressed as

$$
\hat{\sigma}_{i j}=\frac{1}{V} \int_{V} \sigma_{i j} \mathrm{~d} V=\frac{1}{V} \int_{S} \sigma_{i k} x_{j} n_{k} \mathrm{~d} S=\frac{1}{V} \int_{S} T_{i} x_{j} \mathrm{~d} S
$$

where $V$ is the volume of the unit cell $\left(V=L_{x} L_{y} L_{z}\right), n$ is the surface normal, and $T$ is the surface traction. Combining this equation with the condition of stress periodicity yields the following relationships:

$$
\begin{aligned}
& \hat{\sigma}_{x x}=\frac{1}{V}\left(R_{f x} L_{x}-R_{m x} L_{y}\right) \\
& \hat{\sigma}_{x y}=\frac{1}{V} R_{m x} L_{y}
\end{aligned}
$$

Table 1

Parameters used for FE simulations of cracked unit cell.

\begin{tabular}{lcccrrr}
\hline Simulation & $V_{f}$ & $L_{x} / d$ & $E_{f}(\mathrm{GPa})$ & $E_{m}(\mathrm{GPa})$ & $\tau_{s}(\mathrm{MPa})$ & $\hat{p}(\mathrm{MPa})$ \\
\hline Base simulation & 0.4 & 10 & 210 & 100 & 10 & 50 \\
Other & 0.2 & 15 & 100 & 37,400 & 5 \\
\hline
\end{tabular}

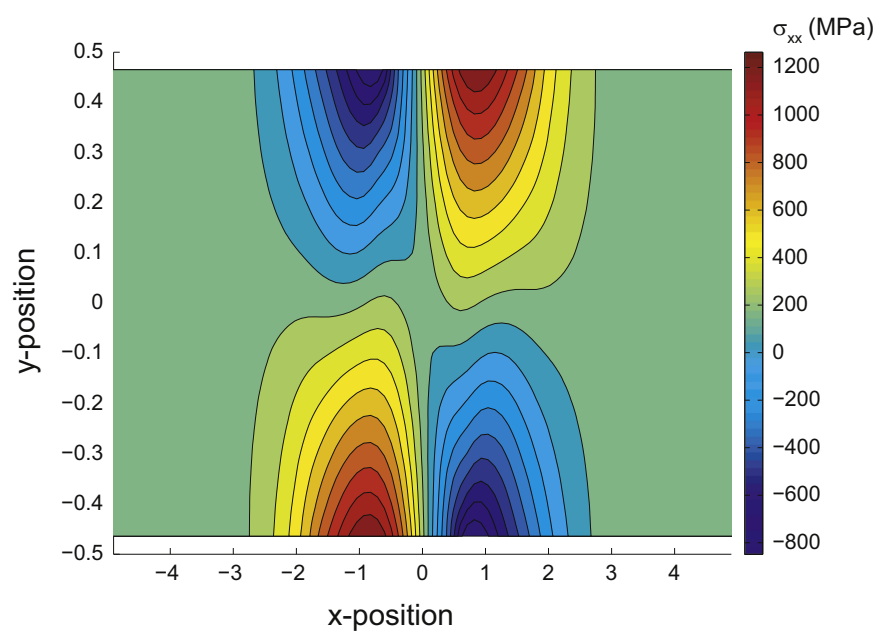

Fig. 7. Axial stress $\left(\sigma_{x x}\right)$ in the fiber at $z=0$ for the baseline simulation of the cracked unit cell $\left(\tau_{a p p}=100 \mathrm{MPa}\right)$. 


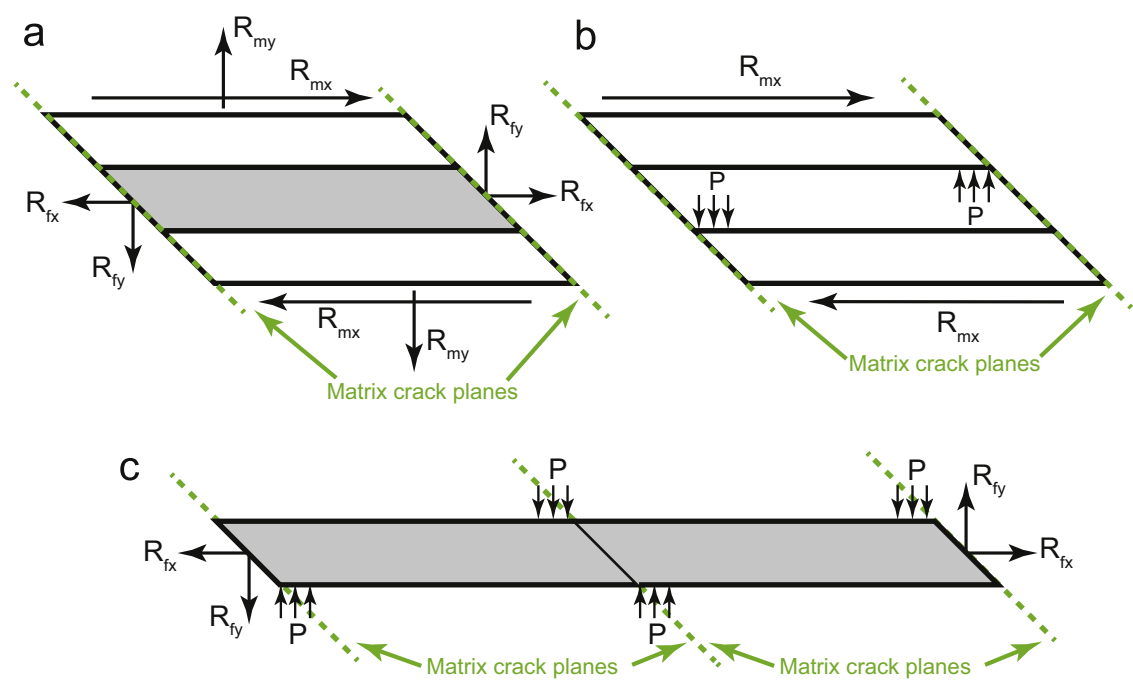

Fig. 8. Free body diagrams for (a) the entire unit cell, (b) the matrix jacket, and (c) the fiber for the cracked composite.

$$
\begin{aligned}
& \hat{\sigma}_{y x}=\frac{1}{V}\left(R_{f y} L_{x}-R_{m y} L_{y}\right) \\
& \hat{\sigma}_{y y}=\frac{1}{V} R_{m y} L_{y}
\end{aligned}
$$

Solving (with $\hat{\sigma}_{x y}=\hat{\sigma}_{y x}$ ),

$$
\begin{aligned}
& R_{f x}=\left(\hat{\sigma}_{x x}+\hat{\sigma}_{x y}\right) L_{y} L_{z} \\
& R_{m x}=\hat{\sigma}_{x y} L_{x} L_{z} \\
& R_{f y}=\left(\hat{\sigma}_{y y}+\hat{\sigma}_{x y}\right) L_{y} L_{z} \\
& R_{m y}=\hat{\sigma}_{y y} L_{x} L_{z}
\end{aligned}
$$

For shear loading, $\hat{\sigma}_{x x}=\hat{\sigma}_{y y}=0$ and $\hat{\sigma}_{x y}=\hat{\sigma}_{y x}=\tau_{a p p}$. Therefore, the force resultants become

$$
\begin{aligned}
& R_{f x}=\tau_{a p p} L_{y} L_{z} \\
& R_{m x}=\tau_{a p p} L_{x} L_{z} \\
& R_{f y}=\tau_{a p p} L_{y} L_{z} \\
& R_{m y}=0
\end{aligned}
$$

The net tension arises because $R_{f x}$ is not zero. Rearranging Eq. (25) (using $L_{y}=L_{z}$ and $V_{f}=\pi d^{2} / 4 L_{y}^{2}$ ) reveals that both the average tensile and average shear stresses on the fiber at the matrix crack plane equal $\tau_{\text {app }} / V_{f}$.

Ancillary relationships are established by enforcing mechanical equilibrium for the individual phases (fiber and matrix) of the composite (Fig. 8(b) and (c)). Considering first the matrix, we see that preserving angular equilibrium requires a pressure distribution to develop along the fiber-matrix interface. The results of the finite element simulation indicate that the 'contact patch' between fiber and matrix is confined to a small region around the matrix crack of length $\approx(1-1.5) d$ (in the $x$-direction). This is indicated schematically by a distributed load in Fig. 8(b). The fiber must be subjected to a distributed load of equal magnitude and of opposite sign. Inspection of Fig. 8(c) clearly reveals that local bending of the fiber in the vicinity of the matrix crack will result.

Note further that the traction applied to the fiber at the crack plane is oriented parallel to the crack plane normal. (This is true regardless of the sign of the applied shear stress.) Such a traction acts against the direction that the fiber is tilted, causing the fiber to rotate into alignment with the crack plane normal. This type of deformation has been termed 'against the nap' by previous studies of bridging of cracks by inclined fibers (Cox and Sridhar, 2002). 'With the nap' deformation of the fiber cannot arise in the problem under present consideration: it would require a different relationship between the orientations of the traction vector, the fiber axis, and the crack plane normal.

\subsection{Fiber stress distribution}

The importance of the various stress components within the fiber can be parsed in an approximate way by considering their relative contributions to the total elastic strain energy. This analysis (which neglects the Poisson effect) reveals that, for the unit cell with crack spacing $L_{x} / d=10, \sigma_{x x}$ accounts for $75-80 \%$ of the strain energy in the fiber and $\sigma_{x y}$ accounts for an additional $10 \%$. The strain energy from the remaining stress components is mostly confined to a narrow 'core' adjacent to 
the matrix crack, where contact stresses are significant: approximately 95\% of this remaining strain energy lies within a distance $\bar{L}_{y}$ of $\bar{x}=0$.

In light of the preceding results, the dominant stresses within the fiber can be captured by accounting for bending and axial tension in the following way. Shear stresses within the fiber are entirely attributable to bending; these stresses are significant compared to the bending stresses because the length over which bending persists is not very large compared to the fiber diameter (i.e., the Bernoulli-Euler assumption does not hold). Eq. (25) implies, crucially, that both the average shear and the average axial stress in the fiber at $x=0$ scale with $\tau_{a p p} / V_{f}$. Therefore, a suitable normalization (denoted by an overbar) for fiber stresses is $\bar{\sigma}=\sigma V_{f} / \tau_{a p p}$. Positions and other geometrical quantities are normalized by the fiber diameter: e.g. $\bar{x}=x / d, \bar{A}=A / d^{2}=\pi / 4, \bar{I}=I / d^{4}=\pi / 64$, where $x$ is the axial coordinate, $A$ is the area of the fiber, and $I$ is the second moment of area. Two functions are taken as unknown: the normalized moment, $\bar{M}(\bar{x})=M(\bar{x}) V_{f} /\left(\tau_{\text {app }} d^{3}\right)$, and the normalized axial stress, $\bar{\sigma}(\bar{x})=\sigma(\bar{x}) V_{f} / \tau_{a p p}$. Using these normalizations, $\sigma_{x x}$ and $\sigma_{y y}$ within the fiber can be expressed as

$$
\bar{\sigma}_{x x}(\bar{x}, \bar{y})=\frac{V_{f}}{\tau_{a p p}} \sigma_{x x}(\bar{x}, \bar{y})=\frac{\bar{M}(\bar{x})}{\bar{I}} \bar{y}+\bar{\sigma}(\bar{x})
$$

and

$$
\bar{\sigma}_{x y}(\bar{x})=\frac{V_{f}}{\tau_{a p p}} \sigma_{x y}(\bar{x})=\frac{1}{\bar{A}} \frac{\mathrm{d} \bar{M}(\bar{x})}{\mathrm{d} \bar{x}}
$$

where $\sigma_{x y}$ is the average shear stress over the fiber cross-section. The normalized distributions $\bar{M}(\bar{x})$ and $\bar{\sigma}(\bar{x})$, depicted in Figs. 9 and 10, are obtained by fitting the $\sigma_{x x}$ stresses from the FE simulations to the form suggested by Eq. (26). That Eq. (26) describes the stress distribution well can be seen in Fig. 7: at a given value of $x$, the distribution in $\sigma_{x x}$ is approximately linear

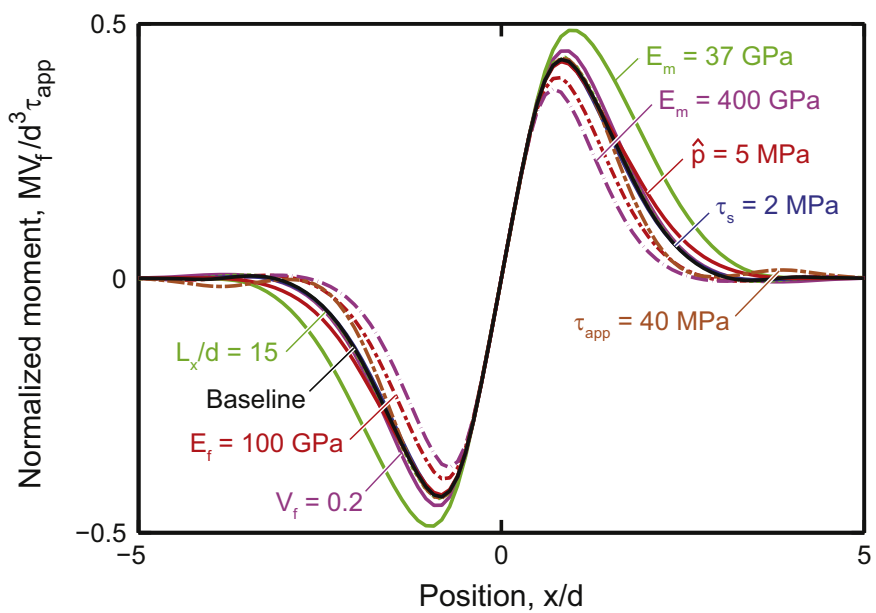

Fig. 9. Normalized moment vs. position for the simulations summarized in Table 1 . (The curves corresponding to $\tau_{S}=2$ MPa and $L_{x} / d=15$ are almost coincident with that corresponding to the baseline simulation.)

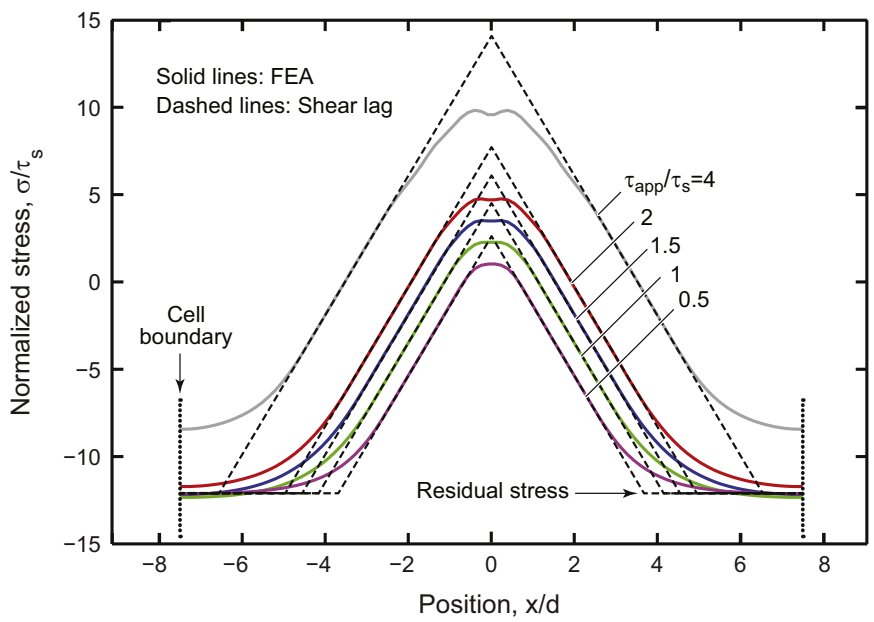

Fig. 10. Normalized axial stress vs. position for the base simulation (with $L_{x} / d=15$ ) with various values of applied stress. 
in $y$. The agreement between $\bar{M}$ obtained from Eq. (26) and that obtained from Eq. (27) (not shown) is also quite good. Taken together, these results indicate that fiber bending and tension are indeed the dominant deformation modes.

The results for $\bar{M}(\bar{x})$ for the base simulation are shown by the solid line in Fig. 9; those for all other simulations (summarized in Table 1) are shown by the dashed lines. Evidently, $\bar{M}(\bar{x})$ is insensitive to the interfacial sliding stress, interfacial pressure, and matrix crack spacing (provided it is large enough that no interaction between matrix cracks occurs), and only weakly sensitive to the ratio of the matrix modulus to fiber modulus. Any effect arising from these variables reflects minor changes in the shape of the pressure distribution at the fiber-matrix contact patch. Furthermore, normalization of the fiber stress by $\tau_{\text {app }} / V_{f}$ essentially eliminates the effects of both $\tau_{a p p}$ and $V_{f}$, consistent with the conclusions drawn from the free body analysis.

Representative numerical results for the axial fiber stress distribution $\bar{\sigma}(\bar{x})$ are shown in Fig. 10 for the base simulation with $L_{x} / d=15$. (Results for the other simulations (not shown) are similar, with exceptions discussed below.) The axial stress in the fiber is largest at $x=0$ and decays away from the matrix crack plane because of frictional slip between the fiber and the matrix. The distribution of axial stress in the fiber can be rationalized using a basic shear-lag model. In this model, the stress decays away from the matrix crack at a rate $\mathrm{d} \sigma / \mathrm{d} x=-4 \tau_{s} / d$ until it reaches its initial (residual) value. The resulting stress distribution is given by

$$
\bar{\sigma}(\bar{x}) \approx \begin{cases}1-\frac{4 \tau_{s} V_{f}}{\tau_{a p p}}|\bar{x}|, & |\bar{x}|<\bar{L}_{a x} \\ \frac{\sigma_{x x}^{f, I} V_{f}}{\tau_{a p p}}, & |\bar{x}| \geq \bar{L}_{a x}\end{cases}
$$

where $\sigma_{x x}^{f, I}$ is the residual stress in the fiber and $\bar{L}_{a x}=L_{a x} / d$ is the normalized axial slip length. Equating the two expressions in Eq. (28) yields the slip length:

$$
\bar{L}_{a x} \approx \frac{\tau_{a p p} / V_{f}-\sigma_{x x}^{f, I}}{4 \tau_{s}}
$$

The predictions of the shear-lag model are in excellent agreement with the FE results over much of the slip zone; discrepancies arise only in the 'core' region (within a distance of about $\pm(1-2) d$ from the crack plane) and in the transition zone to the residual stress. The latter is a consequence of elastic shear transfer where the interfacial shear stress is insufficient for axial slip. (Additional discrepancies arise when the axial slip length exceeds the length of the RVE, but these are merely artifacts of the finite RVE length, selected for computational expediency; the real composite would be essentially infinitely long before the first steady-state matrix crack would emerge and thus there would be no interactions with the boundaries.)

The agreement between the shear-lag model and the FE results breaks down when partial separation between the fiber and the matrix occurs. This behavior is observed in the 'core' region, as well as when as the interfacial pressure, $\hat{p}$, becomes
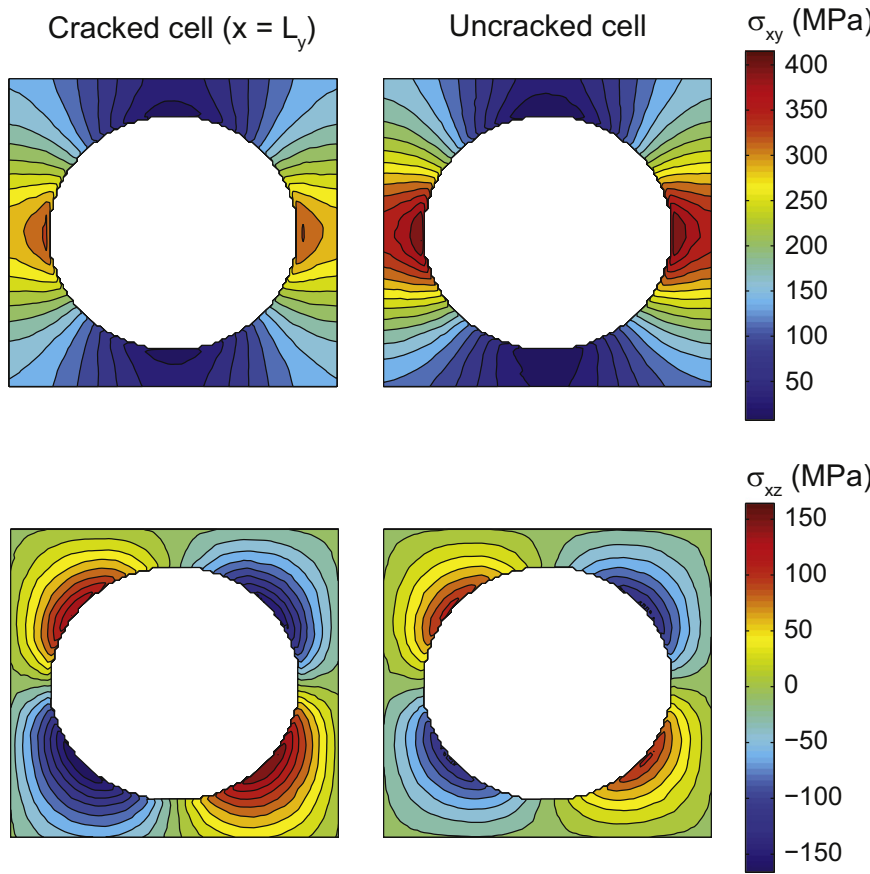

Fig. 11. Contour plots of $\sigma_{x y}$ and $\sigma_{x z}$ from the FE models of the uncracked and cracked unit cells. Results for the cracked unit cell are plotted the edge of the core region $\left(\bar{x}=\bar{L}_{y}\right)$. The applied stress, $\tau_{a p p}=100 \mathrm{MPa}$, is much larger than the interfacial sliding stress $\left(V_{f}=0.4, E_{f}=210 \mathrm{GPa}, E_{m}=100 \mathrm{GPa}, \hat{p}=50 \mathrm{MPa}\right.$, and $\tau_{s}=10 \mathrm{MPa}$ ) 
vanishingly small. If the interface is partially separated, the stress decay occurs at a rate lower than that predicted by the shear-lag model, which is based on full contact. But the results in this limit are not expected to be representative of real composite materials. The reason is that, whereas the model assumes that no shear stresses can be transmitted along the interface for even an infinitesimal separation distance, the finite roughness of real interfaces will enable such transfer even after separation is predicted to occur. We thus argue that the shear-lag model should provide a satisfactory description of the axial stresses even in regions where the computational model predicts partial separation.

\subsection{Matrix stress distribution}

The dominant stress components in the matrix are $\sigma_{x x}, \sigma_{x y}$, and $\sigma_{x z}$; the cumulative strain energy from these components comprises $70-85 \%$ of the total strain energy in the matrix (for $L_{x} / d=10$ ), depending somewhat on the fiber volume fraction. (The contribution is even larger for larger matrix crack spacings.) The axial compressive stress $\sigma_{x x}$ counterbalances the axial tension in the fiber. The shear stresses $\sigma_{x y}$ and $\sigma_{x z}$ arise from shearing of the matrix jacket, and are present even in the uncracked unit cell (Section 4). The remaining stress components are again confined to the 'core' region surrounding the crack: approximately $85 \%$ of the strain energy from $\sigma_{y y}, \sigma_{y z}$, and $\sigma_{z z}$ lies within $\pm \bar{L}_{y}$ of $\bar{x}=0$.

Fig. 11 shows comparisons of the shear stresses $\left(\sigma_{x y}\right.$ and $\left.\sigma_{x z}\right)$ in the matrix of the cracked body at the edge of the core region (at $|\bar{x}|=\bar{L}_{y}$ ) and those in the uncracked body, at an applied stress significantly larger than the interfacial sliding stress. Evidently the matrix shear stresses are almost identical before and after matrix cracking. Moreover, at greater distances from the crack plane $\left(|\bar{x}|>\bar{L}_{y}\right)$, the stresses grow ever closer and become identical in the limit as $|\bar{x}| \rightarrow \infty$. The key implication is that the potential energy difference associated with matrix shear stresses is negligible (apart from that inside the core region).

The axial compressive stress in the matrix can be estimated reasonably well by utilizing the approximate ${ }^{3}$ condition for force equilibrium: $\sigma_{x x}^{f}(\bar{x}) V_{f}+\sigma_{x x}^{m}(\bar{x}) V_{m}=0$. Rearranging this equation yields the matrix stress in terms of the axial tension in the fiber:

$$
\sigma_{x x}^{m}(\bar{x})=-\frac{V_{f}}{V_{m}} \sigma_{x x}^{f}(\bar{x})=-\frac{\tau_{a p p}}{V_{m}} \bar{\sigma}(\bar{x})
$$

In the context of the potential energy change given by Eq. (1), the stress in Eq. (30) represents the only significant contribution from the matrix.

\section{Matrix cracking in shear}

The preceding results for stresses are utilized to determine the matrix cracking stress in the limit of large axial slip lengths. (The same limit was considered by Aveston et al. (1971) in deriving their classical result for the matrix cracking stress in uniaxial tension.) In this limit, the potential energy change arising from stress components confined to the nearcrack ('core') region are neglected. ${ }^{4}$ Furthermore, provided the stress for cracking is sufficiently high to cause slip to occur before cracking, the shear stresses in the constituents outside of the core region are largely unaffected by the presence of the crack and thus the only relevant stress differences, $\sigma^{U}-\sigma^{D}$, derive from the axial stresses $\sigma_{x x}$. The governing equation for steady-state matrix cracking, Eq. (1), becomes

$$
\frac{d}{2} \int_{-\infty}^{\infty}\left[\frac{V_{f}}{E_{f}}\left(\sigma_{x x}^{f, U}-\sigma_{x x}^{f, D}\right)^{2}+\frac{V_{m}}{E_{m}}\left(\sigma_{x x}^{m, U}-\sigma_{x x}^{m, D}\right)^{2}\right] \mathrm{d} \bar{x}=\sqrt{2} V_{m} \mathcal{G}_{m}
$$

Clearly, the upstream axial stresses are simply the residual stresses

$$
\begin{aligned}
& \sigma_{x x}^{f, U}=\sigma_{x x}^{f, I} \\
& \sigma_{x x}^{m, U}=\sigma_{x x}^{m, I}
\end{aligned}
$$

where

$$
V_{f} \sigma_{x x}^{f, I}+V_{m} \sigma_{x x}^{m, I}=0
$$

The downstream axial stresses in the slipped region $\left(|\bar{x}|<\bar{L}_{a x}\right)$ are given approximately by Eqs. (28) and (30). Upon combining the expressions for the upstream and downstream stresses with the governing equation for crack growth (Eq. (31)), we obtain the steady-state matrix cracking stress:

$$
\tau_{c r}=\left(\frac{12 \sqrt{2} V_{f}^{2} V_{m}^{2} E_{f} E_{m} \tau_{s} \mathcal{G}_{m}}{E_{c} d}\right)^{1 / 3}-V_{m} \sigma_{x x}^{m, I}
$$

\footnotetext{
${ }^{3}$ The exact force equilibrium condition involves equilibrium on planes oriented at $45^{\circ}$, as discussed in Section 5.2 . However, the approximate load equilibrium condition is highly accurate outside of the core region.

${ }^{4}$ Note that consideration of these energies would reduce the estimate of the matrix cracking stress.
} 
For comparison, the tensile cracking stress in the absence of residual stress, $\sigma_{1}^{0}$, originally derived by Aveston et al. (1971), is

$$
\sigma_{1}^{0}=\left(\frac{12 V_{f}^{2} E_{f} E_{c}^{2} \tau_{s} \mathcal{G}_{m}}{V_{m} E_{m}^{2} d}\right)^{1 / 3}
$$

Combining with Eq. (34) yields

$$
\tau_{c r}=\frac{2^{1 / 6} V_{m} E_{m}}{E_{c}} \sigma_{1}^{0}-V_{m} \sigma_{x x}^{m, I}
$$

This equation represents one of the key results of the present work. In the absence of residual stress, the ratio of cracking stresses in shear and in tension is given by

$$
\frac{\tau^{c r, 0}}{\sigma_{1}^{0}}=\frac{2^{1 / 6} V_{m} E_{m}}{E_{c}}
$$

To provide further insight into Eq. (37), it is useful to rewrite Eq. (1) in terms of the slip length. It can be shown that this equation reduces to

$$
\begin{aligned}
& \frac{16 d \tau_{s}^{2} V_{f} E_{c}}{3 E_{f} E_{m} V_{m}} \bar{L}_{s, \text { tens }}^{3}=V_{m} \mathcal{G}_{m} \quad \text { (tension) } \\
& \frac{16 d \tau_{s}^{2} V_{f} E_{c}}{3 E_{f} E_{m} V_{m}} \bar{L}_{s, \text { shear }}^{3}=\sqrt{2} V_{m} \mathcal{G}_{m} \quad \text { (shear) }
\end{aligned}
$$

Dividing and rearranging,

$$
\bar{L}_{s, \text { shear }}=2^{1 / 6} \bar{L}_{s, \text { tens }}
$$

when cracking occurs. In the absence of residual stresses,

$$
\begin{aligned}
& \bar{L}_{s, \text { shear }}=\frac{\tau_{a p p}}{4 \tau_{s} V_{f}} \\
& \bar{L}_{s, \text { tens }}=\frac{\sigma_{a p p}}{4 \tau_{s} V_{f}} \frac{V_{m} E_{m}}{E_{c}}
\end{aligned}
$$

Taken together, Eqs. (39) and (40) imply that the difference between cracking stresses in tension and in shear arises from the difference in the respective slip lengths. The slip length for applied tensile loading is proportional to the relative matrix modulus, $V_{m} E_{m} / E_{c}$, whereas that for applied shear loading is not. For equal applied loads, and zero residual stresses, the slip length in tension will always be shorter than that in shear (since $V_{m} E_{m} / E_{c}$ must be less than unity). Therefore, the cracking stress in shear must be smaller than that in tension, by a ratio roughly equal to $V_{m} E_{m} / E_{c}$. For typical values of constituent properties, this ratio equals $0.2-0.6$.

\section{Matrix cracking stress for arbitrary in-plane loading}

The preceding results for matrix cracking in shear and in tension are extended to cracking under arbitrary in-plane loadings, again subject to the assumptions of large-scale slip and of slip preceding matrix cracking. Two further assumptions are made. (i) The matrix crack normal is oriented an angle $\theta$ to the fiber axis. (ii) The normal stress transverse to the fiber axis does not affect the cracking stress. The latter assumption is justified on the basis that this stress component does not alter the axial stresses in the fiber or the matrix. This assumption also implies that the transverse stress does not alter the interfacial constitutive behavior (e.g. by causing interfacial separation). A free-body analysis of a unit cell with a crack oriented at $\theta$ (analogous to that in Section 5.2) shows that the average fiber stress at the crack plane is given by

$$
\sigma_{x x}^{f}(0)=\frac{\sigma_{a p p}+\tau_{a p p} \tan \theta}{V_{f}}
$$

where $\sigma_{\text {app }}$ and $\tau_{\text {app }}$ are the applied normal and shear stresses, respectively. The governing equation for steady-state matrix cracking, Eq. (1), becomes

$$
\frac{d}{2} \int_{-\infty}^{\infty}\left[\frac{V_{f}}{E_{f}}\left(\sigma_{x x}^{f, U}-\sigma_{x x}^{f, D}\right)^{2}+\frac{V_{m}}{E_{m}}\left(\sigma_{x x}^{m, U}-\sigma_{x x}^{m, D}\right)^{2}\right] \mathrm{d} \bar{x}=V_{m} \mathcal{G}_{m} \sec \theta
$$

where the term sec $\theta$ accounts for the area associated with an inclined crack. The upstream axial stresses are given simply by the elastic solution (incorporating residual stresses):

$$
\begin{aligned}
& \sigma_{x x}^{f, U}=\frac{E_{f} \sigma_{a p p}}{E_{c}}+\sigma_{x x}^{f, I} \\
& \sigma_{x x}^{m, U}=\frac{E_{m} \sigma_{a p p}}{E_{c}}+\sigma_{x x}^{m, I}
\end{aligned}
$$


The downstream axial stresses in the slipped region are given by the shear-lag result (Eqs. (28) and (30), modified using Eq. (41) to account for an arbitrarily oriented crack:

$$
\sigma_{x x}^{f, D}= \begin{cases}\frac{\sigma_{a p p}+\tau_{a p p} \tan \theta}{V_{f}}-4 \tau_{s}|\bar{x}|, & |\bar{x}|<\bar{L}_{a x} \\ \frac{E_{f} \sigma_{a p p}}{E_{c}}+\sigma_{x x}^{f, I}, & |\bar{x}| \geq \bar{L}_{a x}\end{cases}
$$

and

$$
\sigma_{x x}^{m, D}(\bar{x})=\frac{\sigma_{a p p}-V_{f} \sigma_{x x}^{f, D}(\bar{x})}{V_{m}}
$$

We assume proportional loading, characterized by $\lambda=\sigma_{\text {app }} / \tau_{\text {app }}$. Upon substituting the expressions for the upstream and downstream stresses (Eqs. (43)-(45)) into the governing equation for crack growth, we obtain the steady-state cracking stresses $\sigma_{a p p}^{c r}$ and $\tau_{a p p}^{c r}$ :

$$
\begin{aligned}
\sigma_{a p p}^{c r} & =\frac{\lambda V_{m} E_{m} \sigma_{1}^{0}(\sec \theta)^{1 / 3}-\lambda V_{m} E_{c} \sigma_{x x}^{m, I}}{E_{c} \tan \theta+\lambda V_{m} E_{m}} \\
\tau_{a p p}^{c r} & =\frac{V_{m} E_{m} \sigma_{1}^{0}(\sec \theta)^{1 / 3}-V_{m} E_{c} \sigma_{x x}^{m, I}}{E_{c} \tan \theta+\lambda V_{m} E_{m}}
\end{aligned}
$$

In the limit of uniaxial tension $(\lambda \rightarrow \infty, \theta=0)$, Eq. (46) reduces to the tensile cracking stress derived by Budiansky et al. (1986):

$$
\sigma^{c r}=\sigma_{1}^{0}-\frac{E_{c}}{E_{m}} \sigma_{x x}^{m, I}
$$

Also, in the limit of pure shear $(\lambda \rightarrow 0, \theta=\pi / 4)$, it reduces to Eq. (36) in the preceding section. An additional case of interest is one in which tension is applied at $45^{\circ}$ to the fiber axis. Here the steady-state cracking stress (again, in the fiber coordinate system) becomes

$$
\sigma_{a p p}^{c r}=\tau_{a p p}^{c r}=\frac{2^{1 / 6} V_{m} E_{m} \sigma_{1}^{0}-V_{m} E_{c} \sigma_{x x}^{m, I}}{E_{c}+V_{m} E_{m}}
$$

so that the $45^{\circ}$ tensile cracking stress is

$$
\sigma_{45}^{c r}=2 \sigma_{a p p}^{c r}=2 \frac{2^{1 / 6} V_{m} E_{m} \sigma_{1}^{0}-V_{m} E_{c} \sigma_{x x}^{m, I}}{E_{c}+V_{m} E_{m}}
$$

In the absence of residual stress, each of these stresses scales with $\sigma_{1}^{0}$. Since $V_{m} E_{m}<E_{c}$, the order of the cracking stresses follows: $\sigma^{c r}>\sigma_{45}^{c r}>\tau^{c r}$.

The results in Eq. (46) can also be expressed in the form of a 'yield surface' in $\sigma-\tau$ space by eliminating $\lambda$. The result is

$$
\sigma_{a p p}^{c r}+\frac{E_{c}}{V_{m} E_{m}} \tau_{a p p}^{c r} \tan \theta=\sigma_{1}^{0}(\sec \theta)^{1 / 3}-\frac{E_{c}}{E_{m}} \sigma_{x x}^{m, I}
$$

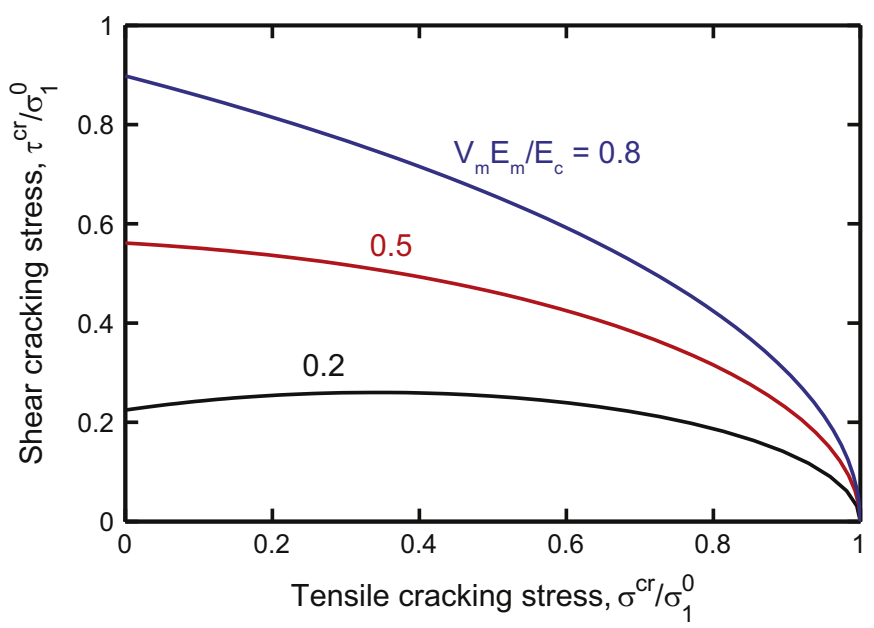

Fig. 12. 'Yield' surfaces (assuming zero residual stress) in $\sigma-\tau$ space, for representative values of relative matrix modulus. 
The form of the yield surface is illustrated in Fig. 12 for representative values of $V_{m} E_{m} / E_{c}$ (absent residual stress). The surfaces are constructed by assuming that the matrix crack is oriented normal to the maximum principal stress. That is, $\theta$ is given by

$$
\theta=\frac{1}{2} \arctan \left(\frac{2 \tau_{a p p}}{\sigma_{a p p}}\right)
$$

where it is assumed that the average stress transverse to the fibers equals zero.

\section{Summary and concluding remarks}

A micromechanical model for the onset of steady-state matrix cracking in unidirectional ceramic composites under shear loading has been developed. The model employs the energy-based criterion for tensile cracking developed by Budiansky et al. (1986) along with the stress fields in upstream and downstream locations. In the limit of large axial slip lengths and when interfacial slip precedes cracking, the shear stresses in the constituents outside of the core region are largely unaffected by the presence of the crack. Instead, the driving force for crack growth derives from the axial stresses-tensile in the fibers and compressive in the matrix-that arise upon cracking. These stresses can be estimated using shear-lag analysis. An analogous approach has been used in deriving the critical stresses needed for matrix cracking under arbitrary in-plane loadings.

We also note that extension of the preceding results on unidirectional plies to composites with multi-directional reinforcement (e.g. cross-ply laminates) requires accounting for stresses induced by the incompatibility between deformations of individual plies. Consider, for instance, a cross-ply laminate loaded in uniaxial tension at $\pm 45^{\circ}$ to the fibers. A continuum-level treatment, in which each ply is treated as a homogeneous, elastic, orthotropic medium, reveals the following effects (Pipes and Pagano, 1970). Each ply experiences not only $\sigma_{45}$, the macroscopic applied tension at $45^{\circ}$, but also a shear stress that arises from the incompatibility of ply deformations. Its magnitude is $C \sigma_{45}$, where $C$ is proportional to the difference between the axial and transverse stiffnesses of an individual ply. However, at a crack (or any free surface) oriented perpendicular to the direction of loading, the shear stress must vanish. Therefore, interlaminar stresses must arise at the intersection of the crack with the inter-ply boundary. These stresses decay rapidly away from the crack, over a length scale proportional to the ply thickness.

To our knowledge, a micromechanical analysis of stresses arising from incompatible ply deformations has yet to be conducted. The preceding continuum-level analysis, however, implies that these stresses can be neglected if either (i) the external state of loading does not give rise to macroscopic ply deformations that are incompatible (a notable example being shear loading); or (ii) the axial and transverse ply stiffnesses are similar, or, equivalently, the fiber and matrix moduli are similar. Therefore, the results presented in Section 6 (on matrix cracking in pure shear) are expected to be applicable to all cross-ply laminates (regardless of constituent properties); conversely, those in Section 7 (on cracking under arbitrary in-plane loads) would be restricted to cross-ply laminates in which the matrix and the fibers exhibit similar stiffness. When the 'incompatibility stresses' are non-negligible, as can be deduced from an analysis similar to that in Pipes and Pagano (1970), they provide another contribution to the crack driving force and thus reduce the estimate of the steady-state matrix cracking stress.

Appropriate characterization of the frictional interaction along the fiber-matrix interface of a CMC remains an outstanding issue (Hutchinson and Jensen, 1990). In the present work, we have considered a rudimentary frictional model: notably, one in which the frictional sliding stress, $\tau_{s}$, is a material constant, independent of the interfacial pressure. Other frictional models, such as Coulomb friction (in which the frictional stress is proportional to the interfacial pressure), are also physically plausible (Hutchinson and Jensen, 1990). Although the two aforementioned models yield similar results for tensile loading (of fibers parallel to the crack plane normal) (Hutchinson and Jensen, 1990), significant differences are expected to arise in the case of shear loading (of fibers inclined with respect to the crack plane normal). Studies of both CMCs (Li et al., 1991) and PMCs (Cartié et al., 2004; Cox, 2006) indicate that the pressure enhancement of the frictional sliding stress (an effect termed 'snubbing') significantly affects the pullout behavior and deformation of the fiber. Further work in this area-that is, investigating the effect of the choice of frictional model on the matrix cracking stress in shear-is clearly required. In this regard, the computational model of the RVE developed in the present work is expected to be highly useful.

Finally, we note that if the composite is unslipped prior to cracking, as is the case for initially bonded interfaces with nonzero debond toughness, crack growth is driven by yet another energy: notably, that resulting from the difference in shear stresses in the constituents between the unslipped and the slipped states. This energy is proportional to the length, $L_{a n t}$, over which antisymmetric slip persists after cracking. In this scenario, the crack growth resistance must also incorporate the debond toughness. This contribution is proportional to the debond length, which is expected to equal $L_{\text {ant }}$. Derivation of the steady-state matrix cracking stress in this scenario would require a fracture mechanics analysis of debonding for off-axis loading, extending the work of Hutchinson and Jensen (1990) on the mechanics of debonding in uniaxial tension.

\section{Acknowledgments}

This work was supported by the Pratt \& Whitney Center of Excellence at the University of California, Santa Barbara (monitored by Douglas Berczik). V.P.R. was supported in part by a National Defense Science and Engineering Graduate Fellowship. 
We acknowledge support from the Center for Scientific Computing at the CNSI and MRL: an NSF MRSEC (DMR-1121053) and NSF CNS-0960316. We also greatly acknowledge fruitful discussions with Matthew Begley and John Hutchinson regarding this work.

\section{References}

Aveston, J., Cooper, G.A., Kelly, A., 1971. Single and multiple fracture. In: The Properties of Fiber Composites, National Physical Laboratory. IPC Science and Technology Press Ltd., Teddington, UK, pp. 15-24.

Beyerle, D.S., Spearing, S.M., Evans, A.G., 1992a. Damage mechanisms and the mechanical properties of a laminated 0/90 ceramic/matrix composite. J. Am. Ceram. Soc. 75, 3321-3330.

Beyerle, D.S., Spearing, S.M., Zok, F.W., Evans, A.G., 1992b. Damage and failure in unidirectional ceramic matrix composites. J. Am. Ceram. Soc. 75, $2719-2725$.

Brøndsted, P., Heredia, F.E., Evans, A.G., 1994. In-plane shear properties of 2-D ceramic matrix composites. J. Am. Ceram. Soc. 77, $2569-2574$.

Budiansky, B., Hutchinson, J.W., Evans, A.G., 1986. Matrix fracture in fiber-reinforced ceramics. J. Mech. Phys. Solids 34, 167-189.

Cady, C., Heredia, F.E., Evans, A.G., 1995. In-plane mechanical properties of several ceramic-matrix composites. J. Am. Ceram. Soc. 78, $2065-2078$.

Cartié, D.D.R., Cox, B.N., Fleck, N.A., 2004. Mechanisms of crack bridging by composite and metallic rods. Composites A 35, $1325-1336$.

Cox, B.N., 2006. Snubbing effects in the pullout of a fibrous rod from a laminate. Mech. Adv. Mater. Struct. 12, 85-98.

Cox, B.N., Sridhar, N., 2002. A traction law for inclined fiber tows bridging mixed-mode cracks. Mech. Adv. Mater. Struct. 9, $299-331$.

Curtin, W.A., 1991. Theory of mechanical properties of ceramic-matrix composites. J. Am. Ceram. Soc. 74, 2837-2845.

Evans, A.G., Domergue, J.M., Vagaggini, E., 1994. Methodology for relating the tensile constitutive behavior of ceramic-matrix composites to constituent properties. J. Am. Ceram. Soc. 77, 1425-1435.

Evans, A.G., Zok, F.W., 1994. The physics and mechanics of fibre-reinforced brittle matrix composites. J. Mater. Sci. 29, 3857-3896.

Genin, G.M., Hutchinson, J.W., 1997. Composite laminates in plane stress: constitutive modeling and stress redistribution due to matrix cracking. J. Am. Ceram. Soc. 80, 1245-1255.

Hashin, Z., 1983. Analysis of composite materials-a survey. J. Appl. Mech. 50, 481-505.

Hashin, Z., Rosen, B.W., 1964. The elastic moduli of fiber-reinforced materials. J. Appl. Mech. 31, 223-232.

Hui, C.Y., Phoenix, S.L., Ibnabdeljalil, M., Smith, R.L., 1995. An exact closed form solution for fragmentation of Weibull fibers in a single filament composite with applications to fiber-reinforced ceramics. J. Mech. Phys. Solids 43, 1551-1585.

Hutchinson, J.W., Jensen, H.M., 1990. Models of fiber debonding and pullout in brittle composites with friction. Mech. Mater. 9, 139-163.

Li, V.C., Wang, Y., Backer, S., 1991. A micromechanical model of tension-softening and bridging toughening of short random fiber reinforced brittle matrix composites. J. Mech. Phys. Solids 39, 607-625.

Marshall, D.B., Cox, B.N., 1988. A J-integral method for calculating steady-state matrix cracking stresses in composites. Mech. Mater 7, 127-133.

Marshall, D.B., Cox, B.N., Evans, A.G., 1985. The mechanics of matrix cracking in brittle-matrix fiber composites. Acta Metall. 33, $2013-2021$.

Pipes, R.B., Pagano, N.J., 1970. Interlaminar stresses in composite laminates under uniform axial extension. J. Compos. Mater. 4, 538-548.

Spearing, S.M., Zok, F.W., 1993. Stochastic aspects of matrix cracking in brittle matrix composites. J. Eng. Mater. Technol. 115, 314-318.

Suquet, P., 1987. Elements of homogenization theory for inelastic solid mechanics. In: Sanchez-Palencia, E., Zaoui, A. (Eds.), Homogenization Techniques for Composite Media. Springer-Verlag, Berlin, pp. 194-275.

Totry, E., Molina-Aldareguìa, J.M., González, C., LLorca, J., 2010. Effect of fiber, matrix, and interface properties on the in-plane shear deformation of carbonfiber reinforced composites. Compos. Sci. Technol. 70, 970-980.

Turner, K.R., Speck, J.S., Evans, A.G., 1995. Mechanisms of damage and failure in carbon-matrix composites subject to tensile and shear loading. J. Am. Ceram. Soc. 78, 1841-1848.

Xia, Z., Zhang, Y., Ellyin, F., 2003. A unified periodical boundary conditions for representative volume elements of composites and applications. Int. J. Solids Struct. 40, 1907-1921.

Xia, Z.C., Carr, R.R., Hutchinson, J.W., 1993. Transverse cracking in fiber-reinforced, brittle matrix cross-ply composites. Acta Metall. Mater. 41, $2365-2376$.

Xia, Z.C., Hutchinson, J.W., 1994. Matrix cracking of cross-ply ceramic composites. Acta Metall. Mater. 42, 1933-1945.

Zok, F.W., Spearing, S.M., 1992. Matrix crack spacing in brittle matrix composites. Acta Metall. Mater. 40, 2033-2043. 